\title{
The relationship between admission blood glucose levels and hospital mortality
}

\author{
N. W. Cheung $\cdot \mathrm{S}$. Li $\cdot$ G. Ma $\cdot$ R. Crampton
}

Received: 13 December 2007 / Accepted: 17 March 2008/Published online: 16 April 2008

(C) Springer-Verlag 2008

\begin{abstract}
Aims/hypothesis The purpose of this study was to examine the relationship between blood glucose level (BGL) on admission with mortality rates among patients admitted to hospital through the Emergency Department.

Methods In a prospective observational study, BGLs were routinely measured on 6,187 consecutive patients requiring blood testing and admitted through the Emergency Department of a tertiary referral hospital. These measurements were matched against demographic data and hospital mortality rates.

Results Overall in-hospital mortality was $4.8 \%$. Admission BGL was an independent predictor of mortality (HR 1.04 per $1 \mathrm{mmol} / 1$ increase, 95\% CI 1.02-1.06, $p=0.02$ ). There was a significant interaction between diabetes status and increasing BGL on mortality $(p<0.001)$, with higher BGLs being associated with greater mortality among non-diabetic than among diabetic patients. Among non-diabetic patients, the lowest mortality rate $(3.0 \%)$ was in people with a BGL of 4.0-5.9 mmol/l. Compared with this group, patients with a BGL of $8.0-9.9 \mathrm{mmol} / \mathrm{l}$ had increased mortality rate
\end{abstract}

N. W. Cheung $(\bowtie)$

Centre for Diabetes and Endocrinology Research,

Westmead Hospital,

Hawkesbury Road,

Westmead, NSW 2145, Australia

e-mail:wah@westgate.wh.usyd.edu.au

S. Li • G. Ma

Institute of Clinical Pathology and Medical Research,

Westmead Hospital,

Westmead, NSW, Australia

R. Crampton

Emergency Department, Westmead Hospital,

Westmead, NSW, Australia
(7.6\%, HR 1.56, 95\% CI 1.03-2.35, $p=0.04$, after adjustment for age and sex). The risk increased further at higher glucose levels. In the cohort with diagnosed diabetes, the increase in mortality rates at higher BGL bands was not significant.

Conclusions/interpretation Among people who do not have diabetes, even modest degrees of hyperglycaemia on hospital admission are associated with increased mortality.

Keywords Diabetes $\cdot$ Glucose $\cdot$ Hospital $\cdot$ Hyperglycaemia . Mortality

\section{Abbreviation \\ BGL blood glucose level}

\section{Introduction}

Hyperglycaemia in critical illness is associated with increased mortality. This has been demonstrated in a number of clinical situations, most notably myocardial infarction [1], stroke [2] and intensive care [3]. There are also data from a general hospital indicating that patients with newly diagnosed hyperglycaemia have an increased risk of death [4].

It is unclear, however, at which threshold of blood glucose the risk of mortality increases for hospitalised patients. Most studies have analysed hyperglycaemia as a dichotomised variable [1, 2], but the cut-off levels used do not necessarily best reflect a threshold for increased hospital mortality rates. There is also a paucity of data regarding the nature of the relationship between blood glucose levels (BGLs) and hospital mortality. While a dose-response relationship between BGLs and mortality 


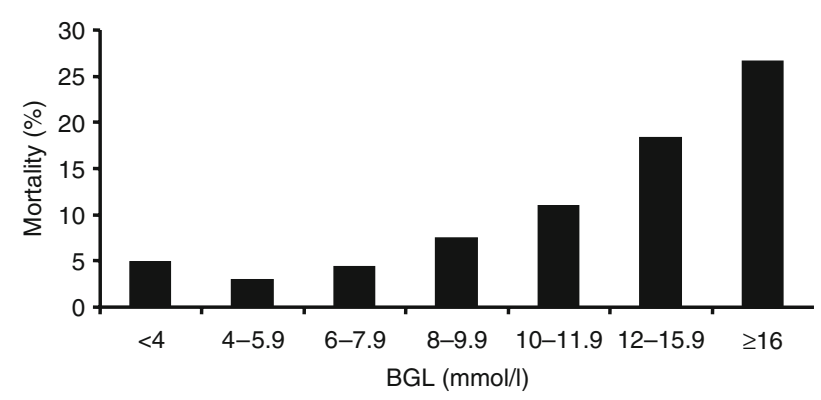

$\begin{array}{lccccccc}\text { People } & 81 & 2,482 & 1,972 & 437 & 118 & 87 & 15 \\ \text { Deceased } & 4 & 74 & 79 & 33 & 13 & 16 & 4 \\ \text { HR } & 1.7 & \text { reference } & 1.1 & 1.6 & 2.6 & 3.5 & 5.3 \\ 95 \% \text { Cl } & 0.6-4.6 & \text { group } & 0.8-1.5 & 1.0-2.4 & 1.4-4.7 & 2.0-6.1 & 1.9-14.7 \\ p \text { value } & 0.32 & & 0.56 & 0.04 & 0.02 & <0.001 & 0.001\end{array}$

Fig. 1 Relationship between mortality and admission BGL for patients without known diabetes. The values below the graph show the HRs with 95\% CIs, using Cox regression, compared with the reference group in the 4.0-5.9 $\mathrm{mmol} / 1 \mathrm{BGL}$ band, after adjustment for age and sex

has been demonstrated in myocardial infarction [5], there are no such data for hospitalised patients in general. To answer these questions, we examined the relationship between BGL on hospital admission and inpatient mortality rates.

\section{Methods}

This was a prospective observational study, conducted between May and November 2003 at Westmead Hospital, a tertiary referral hospital serving a population of approximately one million people. All patients who had a blood test when admitted to hospital through the Emergency Department also had an opportunistic measurement of blood glucose. The results were displayed in the hospital's computerised pathology reporting system and were freely available to the doctors responsible for the patients' care. The study team did not intervene, except for a small number of patients admitted under the direct care of one of the authors (N. W. Cheung). This study was approved by the hospital Human Research and Ethics Committee.

Glucose concentrations were mostly determined from plasma samples obtained from blood collected in fluoride oxalate sample tubes. In some cases, they were measured from tubes with clot activator. Samples were centrifuged at $900 \mathrm{~g}$ and separated within $30 \mathrm{~min}$, and assayed within 120 min using the coupled hexokinase/glucose-6-phosphate dehydrogenase method and the Roche/Hitachi Modular P system (Roche Diagnostics, Basel, Switzerland).

The results of the admission BGL were matched against data from the hospital administrative database, which included International Statistical Classification of Diseases, 10th revision, 2003 version (ICD-10; available from http:// www.who.int/classifications/apps/icd/icd10online2003/ fr-icd.htm, accessed 24 March 2008) coding (from which the presence of diabetes was determined), demographic details, mode of separation (including death) and length of stay or time to death. Statistics were analysed by the program SPSS (SPSS, Chicago, IL, USA). Mortality analyses were based on time to death, using Cox regression models. Adjustments were made for age and sex, as stated.

\section{Results}

Blood glucose levels were obtained from blood tests, which were available for 6,187 (3,147 men, 3,040 women) of the 7,408 consecutive patients admitted during the study period. Among people whose BGL was measured, the mean age was $59.1 \pm 21.2$ years. There were 1,175 people $(19.0 \%)$ with known diabetes. A total of 295 deaths occurred in hospital (4.8\%). People who did not have BGL measured had a similar mortality rate and length of stay, but were younger and less likely to have diabetes. Admission BGL was an independent predictor of mortality (HR 1.04 per $1 \mathrm{mmol} / \mathrm{l}$ increase, 95\% CI 1.02-1.06, $p=0.02$ ), as was age (HR $1.03,95 \%$ CI $1.03-1.04$, $p<0.001$ ) and sex (males 5.0\%, females $4.6 \%$, HR 1.28 , $95 \%$ CI $1.02-1.62, p=0.04)$. The presence of diabetes was not in itself associated with increased mortality risk (6.1 vs $4.4 \%$, HR 1.29, 95\% CI 0.94-1.76, $p=0.11)$. However, there was a statistically significant interaction between the effect of diabetes and increasing BGL on mortality rate $(p<0.001)$. In subsequent analyses, therefore, people with and without diabetes were examined separately.

To determine a glucose threshold for increased mortality, people were divided according to their BGL on admission, using 2-4 mmol/1 increments as the cut-off levels to define BGL bands (Figs 1 and 2). Among patients without diabetes, the lowest mortality rate was in the 4.0
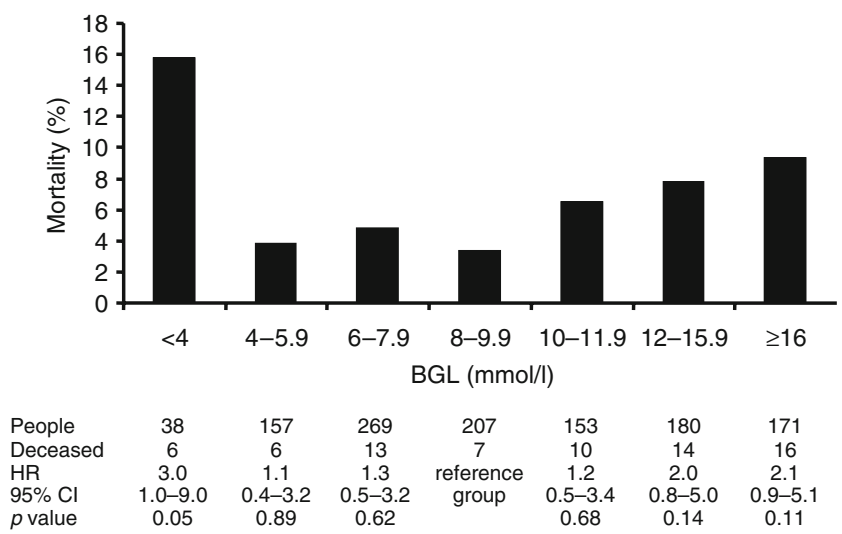

Fig. 2 Relationship between mortality and admission BGL for patients with known diabetes. The values below the graph show the HRs with 95\% CIs, using Cox regression, compared with the reference group in the $8.0-9.9 \mathrm{mmol} / 1$ BGL band, after adjustment for age and sex 
$5.9 \mathrm{mmol} / \mathrm{l} \mathrm{BGL}$ band (3.0\%). Using this BGL band as the reference group for comparisons (Fig. 1), an increased mortality rate was found in the $8.0-9.9 \mathrm{mmol} / \mathrm{l} \mathrm{BGL}$ band (7.6\%), even after adjustment for age and sex (HR 1.56, $95 \%$ CI $1.03-2.35, p=0.04)$. The risk of death rose further in the higher BGL bands, peaking at $26.7 \%$ among individuals with a $\mathrm{BGL} \geq 16 \mathrm{mmol} / \mathrm{l}$ (adjusted HR 5.28, 95\% CI 1.90-14.7, $p=0.001$ ).

As increased mortality rates were seen above a BGL of $8.0 \mathrm{mmol} / \mathrm{l}$, further analysis was conducted with BGL as a dichotomised variable using this cut-off level. The admission $\mathrm{BGL}$ was $\geq 8.0 \mathrm{mmol} / 1$ in $13.1 \%$ of patients without diagnosed diabetes. Compared with patients whose BGL upon admission was $<8.0 \mathrm{mmol} / \mathrm{l}$, mortality rate was increased among those with a BGL $\geq 8.0 \mathrm{mmol} / 1$ (HR $1.98,95 \%$ CI $1.48-2.63, p<0.001$, after adjustment for age and sex).

In the cohort with diagnosed diabetes, the mortality rate was lowest in the 8.0-9.9 mmol/1 BGL band (3.4\%). Using this band as the reference group for comparisons, we found a trend to increased mortality in the higher BGL bands, but these did not reach significance (Fig. 2). However, increased mortality rates were seen among individuals with a BGL $<4.0 \mathrm{mmol} / 1$ (15.8\%), even after adjustment for age and sex (HR 3.01, 95\% CI 1.01-9.00, $p=0.05$ ). Use of the $4.0-5.9 \mathrm{mmol} / \mathrm{l}$ band as reference changed little, except that the increased mortality rate in the $<4.0 \mathrm{mmol} / \mathrm{l}$ BGL band fell out of significance.

\section{Discussion}

This prospective cohort study found that among people without known diabetes, there is a strong association between admission BGL and in-patient mortality, within the entirety of a heterogeneous population admitted through a hospital Emergency Department. From the analysis of BGL as a categorical variable, increased risk occurred above a BGL of $8.0 \mathrm{mmol} / \mathrm{l}$.

Stress hyperglycaemia, occurring in patients who do not have diabetes, has been associated with greater mortality risk than the presence of diabetes as such [1,2]. Our data concur with this, with the relationship between BGL and mortality rate being considerably stronger in individuals without diabetes. For a given level of hyperglycaemia, nondiabetic people probably have greater severity of illness, which contributes to increased mortality. However, it is possible that hyperglycaemia itself also predisposes to increased mortality. Supporting this, it has been found that hyperglycaemia is associated with increased C-reactive protein and other inflammatory cytokines, increased coagulability and oxidative stress, impairment of leucocyte function and increased rates of infection [6].
Meta-analyses of studies where hyperglycaemia was defined as an admission BGL above the $6.1-8.0 \mathrm{mmol} / 1$ band found that the relative risk of mortality among hyperglycaemic patients who did not have diabetes was 3.9 times for myocardial infarction [1] and 3.07 times for stroke [2]. Many of these studies examined mortality rates with glucose as a dichotomised variable, often based on arbitrary cut-off levels or concentrations relating to the diagnosis of diabetes or impaired glucose tolerance. These cut-off levels do not necessarily best reflect a glucose threshold above which patients are at increased risk. The large number of patients in the current study enabled us to better define a threshold for increased mortality risk. Although the increase in mortality risk with admission glucose levels appears to be linear, when analysed as a categorical variable, it became significant above a BGL of $8.0 \mathrm{mmol} / \mathrm{l}$. With this in mind, and due to the fact that individuals with a $\mathrm{BGL} \geq 8.0 \mathrm{mmol} / 1$ had a doubling of mortality risk, but comprised only $13 \%$ of the non-diabetic patient population, we suggest that a BGL of $8.0 \mathrm{mmol} / \mathrm{l}$ may be an appropriate threshold for defining a minimum glucose level above which increased risk occurs and intervention might be considered.

One limitation of the current study is that some of the hyperglycaemic individuals classified as non-diabetic may well have had undiagnosed diabetes. Additionally, we did not have a record of glycaemic control during the hospital stay. However, patients admitted with hyperglycaemia have been shown to have higher BGLs on subsequent days [7]. Thus, admission hyperglycaemia reflects a tendency towards hyperglycaemia during the hospital stay. It is likely that this longer exposure to hyperglycaemia, rather than the single BGL on admission, contributes to the increased mortality risk. Therefore, one important inference from the association between admission BGL and hospital mortality rates is the possibility that better glucose control during the hospital stay may improve outcomes. Indeed, insulin infusion administered to achieve a BGL below $6.1 \mathrm{mmol} / 1$ has reduced mortality rates among patients in intensive care [8]. The Diabetes Mellitus Insulin-Glucose Infusion in Acute Myocardial Infarction (DIGAMI) study has also demonstrated that peri-infarct administration of insulin to maintain the BGL below $10 \mathrm{mmol} / \mathrm{l}$ reduced mortality [9]. In another study, insulin infusion therapy did not improve survival among hyperglycaemic patients with myocardial infarction [10]. However, the 6 month mortality rate was only $2 \%$ among patients who maintained a mean BGL $\leq 8.0 \mathrm{mmol} / 1$ in the first $24 \mathrm{~h}$ compared with $11 \%$ among patients with a mean $\mathrm{BGL}>8.0 \mathrm{mmol} / \mathrm{l}$.

The above intervention studies indicate that tight glucose control is likely to be beneficial in critically ill patients. It is as yet unknown whether this would also be the case for general hospital patients without diabetes. Nonetheless, given the strong relationship between admission BGL and 
mortality, we suggest that control of hyperglycaemia in hospitalised patients may be of therapeutic value. Randomised clinical trials should be performed to establish its benefit.

Acknowledgements We thank F. Heydari, J. Youssef and Y. Sader for their work on the database, and $\mathrm{K}$. Byth for the statistical analyses. This study was funded through a Novo-Nordisk Regional Diabetes Support Scheme Grant.

Duality of interest The authors declare that there is no duality of interest associated with this manuscript.

\section{References}

1. Capes SE, Hunt D, Malmberg K, Gerstein HC (2000) Stress hyperglycaemia and increased risk of death after myocardial infarction in patients with and without diabetes: a systematic overview. Lancet 355:773-778

2. Capes SE, Hunt D, Malmberg K, Pathak P, Gerstein HC (2001) Stress hyperglycaemia and prognosis of stroke in nondiabetic and diabetic patients: a systematic overview. Stroke 32:2426-2432

3. Finney SJ, Zekveld C, Elia A, Evans TW (2003) Glucose control and mortality in critically ill patients. JAMA 290:2041-2047
4. Umpierrez GE, Isaacs SD, Bazargan N, You X, Thaler LM, Kitabchi AE (2002) Hyperglycemia: an independent marker of in-hospital mortality in patients with undiagnosed diabetes. J Clin Endocrinol Metab 87:978-982

5. Wong V, Ross DL, Park K, Boyages S, Cheung NW (2004) Hyperglycemia following acute myocardial infarction is a predictor of poor cardiac outcomes in the reperfusion era. Diab Res Clin Pract 64:85-91

6. Clement S, Braithwaite SS, Magee MF et al (2004) Management of diabetes and hyperglycemia in hospitals. Diabetes Care 27:553-590

7. Cely CM, Arora P, Quartin AA, Kett DH, Schein RMH (2004) Relationship of baseline glucose homeostasis to hyperglycemia during medical critical illness. Chest 126:879-887

8. Van den Berghe G, Wouters P, Weekers F et al (2001) Intensive insulin therapy in critically ill patients. N Engl J Med 345:13591367

9. Malmberg K, Ryden L, Efendic S et al (1995) Randomised trial of insulin-glucose infusion followed by subcutaneous insulin treatment in diabetic patients with acute myocardial infarction (DIGAMI Study): effects on mortality at 1 year. J Am Coll Cardiol 26:57-65

10. Cheung NW, Wong VW, McLean M (2006) The Hyperglycemia: intensive insulin infusion in infarct (HI-5) study - a randomised controlled trial of insulin infusion therapy for myocardial infarction. Diabetes Care 29:765-70 